\title{
Evaluation of corneal endothelium and keratic precipitates by specular microscopy in anterior uveitis
}

\author{
C T Pillai, H S Dua, A Azuara-Blanco, A R Sarhan
}

\begin{abstract}
Background-The study of corneal endothelium, by specular microscopy, in patients with anterior uveitis has largely been restricted to observations on the endothelial cells. In this prospective study "keratic precipitates" (KP) in different types of uveitis were examined in different stages of the disease process and the endothelial changes occurring in the vicinity of the KP were evaluated in comparison with the endothelium of the uninvolved eye.
\end{abstract}

Methods-13 patients with active unilateral uveitis were recruited. The mean age was 42.9 years (range 20-76 years). A Tomey-1100 contact wide field specular $(\times 10)$ microscope was used to capture endothelial images and KP until the resolution of uveitis. Data regarding type of uveitis, number, size, and nature of KP were recorded. Automated morphometric analysis was done for cell size, cell density and coefficient of variation, and statistical comparisons of cell size and cell density were made (Student's $t$ test) between the endothelium in the vicinity of fresh and resolving KP, fresh $\mathrm{KP}$ and normal endothelium, and resolving $\mathrm{KP}$ and normal endothelium.

Results-On specular microscopy, fresh KP were seen as dense, white glistening deposits occupying 5-10 endothelial cells in diameter and fine KP were widely distributed and were one or two endothelial cells in diameter. The KP in PosnerSchlossman syndrome had a distinct and different morphology. With clinical remission of uveitis, the KP were observed to undergo characteristic morphological changes and old KP demonstrated a large, dark halo surrounding a central white deposit and occasionally a dark shadow or a "lacuna" replaced the site of the original
KP. Endothelial blebs were noted as dark shadows or defects in the endothelial mosaic in patients with recurrent uveitis. There was significant statistical difference in the mean cell size and cell density of endothelial cells in the vicinity of fresh KP compared with normal endothelium of the opposite eye.

Conclusion-This study elucidated the different specular microscopic features of $\mathrm{KP}$ in anterior uveitis. Distinct morphological features of large and fine KP were noted. These features underwent dramatic changes on resolution of uveitis. The endothelium was abnormal in the vicinity of $\mathrm{KP}$, which returned to near normal values on resolution of uveitis.

(Br f Ophthalmol 2000;84:1367-1371)

Specular microscopy enables us to study the changes occurring in different layers of cornea in various anterior segment disorders at much higher magnification. Since its invention 20 years ago, many technological advances have been made in the design and application of this technique. ${ }^{1-5}$ With the modern specular microscopes, endothelial images can be digitised and automated morphometric analysis of endothelial cells can be done. ${ }^{6-9}$

Anterior segment inflammation causes changes in corneal endothelium and these changes have been observed by specular microscopy in various studies. ${ }^{10-16}$ Inflammatory changes affecting the endothelium can be serious enough to affect the endothelial integrity and that of cornea itself, particularly the capacity of the endothelium to withstand operative intervention. ${ }^{10}{ }^{11}$ Polymorphism and polymegathism of endothelial cells can occur in anterior uveitis. In this prospective study, we observed and analysed the appearance of keratic precipitates (KP) in different types of uveitis and at different stages of disease
Department of Ophthalmology, B Floor, South Block, University Hospital, Queen's Medical Centre, Nottingham NG7 2UH, UK

C T Pillai

H S Dua

A Azuara-Blanco

A R Sarhan

Correspondence to: Professor Harminder S Dua harminder.dua@nottingham. ac.uk

Accepted for publication 23 May 2000
Table 1 Characteristics of keratic precipitates (KP)

\begin{tabular}{llllll}
\hline Number & Sex & Age & Diagnosis & Type of KP & Number of KP \\
\hline 1 & M & 54 & Acute iritis & Fine, pigmented & $<5$ \\
2 & M & 43 & Posner-Schlossman & Large, pigmented & $<5$ \\
3 & M & 75 & Recurrent iritis & Medium, pigmented & $>15$ \\
4 & F & 40 & Heterochromic cyclitis & Fine, pigmented & Single \\
5 & F & 50 & Retinal vasculitis & Fine, pigmented & $<5$ \\
6 & M & 40 & Acute iritis & Fine, pigmented & $>15$ \\
7 & M & 30 & Herpetic uveitis & Fine, pigmented & $>25$ \\
8 & M & 60 & Recurrent iritis & Medium, non-pigmented & $>5$ \\
9 & F & 63 & Posner-Schlossman & Stellate, medium & Single \\
10 & F & 28 & Acute iritis & Fine, non-pigmented & $>15$ \\
11 & M & 54 & Acute iritis & Medium, non-pigmented & $>15$ \\
12 & M & 52 & Acute iritis & Fine, pigmented & $>10$ \\
13 & M & 76 & Recurrent iritis & &
\end{tabular}


Table 2 Cell density and cell size

\begin{tabular}{lllllll}
\hline & \multicolumn{5}{c}{ Cell density } & \multicolumn{5}{c}{ Cell size } \\
\cline { 2 - 6 } No & Fresh KP & Resolving KP & Normal endothelium & Fresh KP & Resolving KP & Normal endothelium \\
\hline 1 & 2541.5 & 2752.0 & 2558.0 & 401.5 & 363.5 & 390.0 \\
2 & 2172.5 & 2326.0 & 2428.0 & 460.0 & 429.5 & 41.0 \\
3 & 2490.0 & 2350.0 & 2854.0 & 401.0 & 425.5 & 354.0 \\
4 & 2565.5 & 2424.0 & 2974.0 & 389.5 & 424.0 & 336.0 \\
5 & 2347.0 & 2578.5 & 2866.0 & 428.5 & 389.0 & 348.0 \\
6 & 2241.0 & 2729.0 & 2364.0 & 446.0 & 366.0 & 390.0 \\
7 & 2399.0 & 2662.0 & 2558.0 & 428.0 & 375.0 & 375.0 \\
8 & 2361.5 & 2603.0 & 266.0 & 423.0 & 384.0 & 378.0 \\
9 & 2445.5 & 2570.5 & 2492.0 & 409.5 & 390.0 & 373.0 \\
10 & 2796.0 & 2716.5 & 2641.0 & 357.0 & 367.5 & 357.0 \\
11 & 2646.5 & 2530.5 & 2679.0 & 377.0 & 396.0 & 368.0 \\
12 & 2563.5 & 2377.5 & 2800.0 & 389.5 & 420.0 & 385.0 \\
13 & 2664.0 & 2592.0 & 2710.0 & 375.0 & &
\end{tabular}

No $=$ number $; \mathrm{KP}=$ keratic precipitates.

process. Endothelium in the vicinity of the KP was evaluated in comparison with the endothelium of the uninvolved eye. To our knowledge this is the first study which describes the
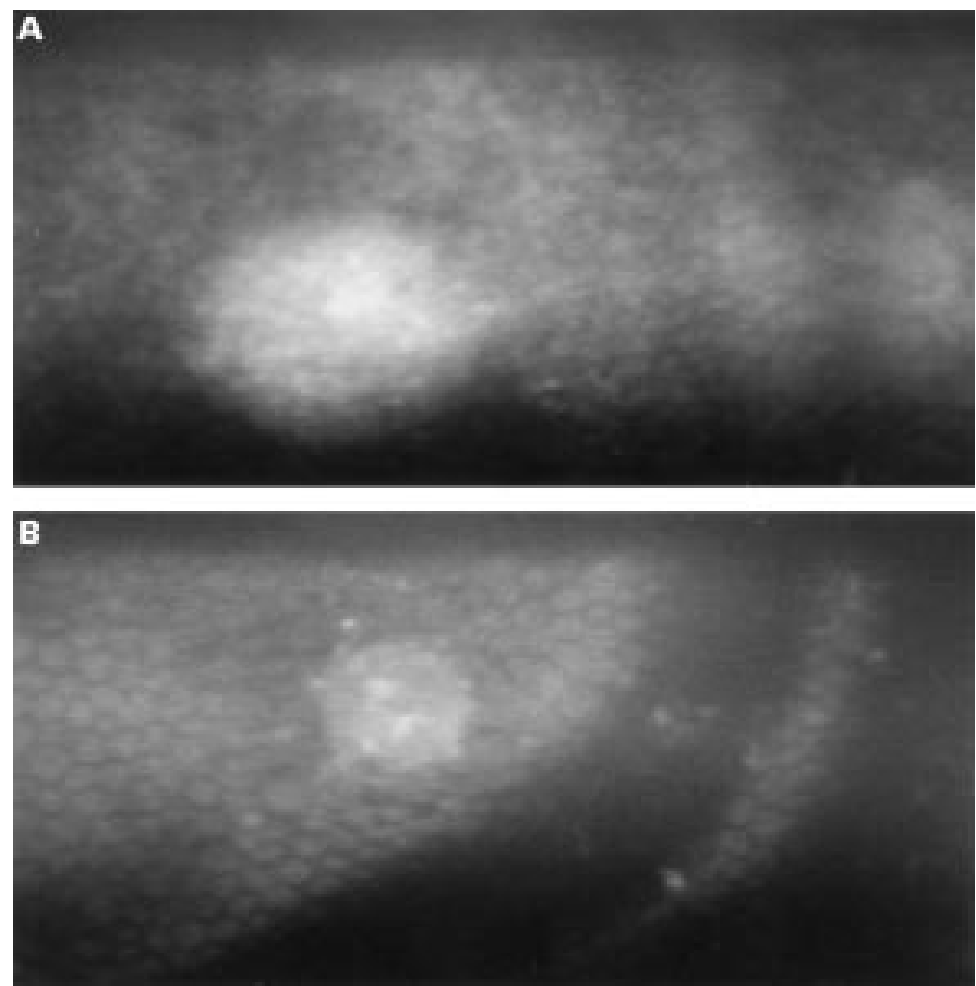

Figure 1 (A) and (B) Large fresh KP appearing as dense white deposits on endothelium.

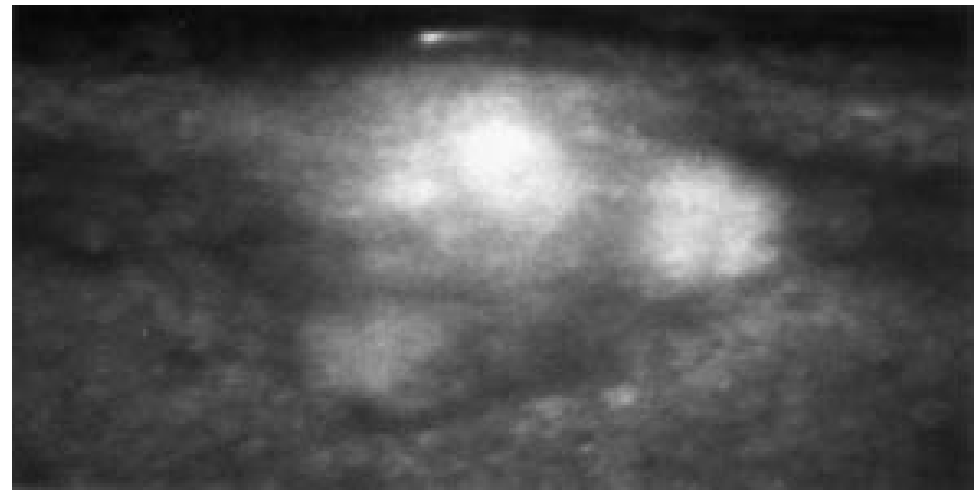

Figure 2 The solitary KP in Posner-Schlossman syndrome. Note the conglomeration of three individual KP connected by slender pseudopodia and the margin of the conglomerate distinct from the surrounding endothelium. distinct morphological features of $\mathrm{KP}$ in different types of uveitis.

\section{Materials and methods}

Patients with active anterior uveitis attending the eye casualty department or the uveitis clinic at Queen's Medical Centre, University Hospital, Nottingham, UK, were recruited for the study. Informed consent was obtained. Patients with previous ocular surgery, trauma, contact lens history, corneal dystrophy, corneal oedema, high intraocular pressure (IOP), and any other anterior segment disorders affecting the corneal endothelium were excluded from the study. Only patients with active unilateral uveitis were included.

On initial examination data concerning age, sex, ocular symptoms, medications, previous surgery, and systemic diseases were recorded. Every patient underwent a complete anterior and posterior segment examination. On slit lamp examination the characteristics of the $\mathrm{KP}$-number, size, (fine or mutton fat), nature of the KP (fresh versus old), pigmentation, and their position on the endothelium-were recorded in a diagrammatic pattern (Table 1). Any other deposits, pigments on the endothelium and other corneal abnormalities were also noted. The location of the KP and deposits were recorded in the four quadrants of the cornea and this diagram was later used to study the KP on the endothelial mosaic by specular microscope. The patients were treated with topical steroids and cyclopentolate $1 \%$ eye drops and followed up every 2 weeks by the same examiner until the resolution of the uveitis.

A Tomey-1100 wide field contact specular microscope was used to capture the endothelial images. Benoxinate $0.4 \%$ drops were used to anaesthetise the eyes and patients were asked to concentrate on the fixation light in the microscope and the contact probe $(\times 10$ magnification) was used to record the pictures. Endothelial images were captured from four different quadrants of the eye with uveitis and also from the normal eye for comparison. After recording, the images with good contrast were selected and stored in the IBM computer. Two rectangles of equal square area were chosen on either side of the KP and automated morphometric analysis was carried out for cell size, cell 


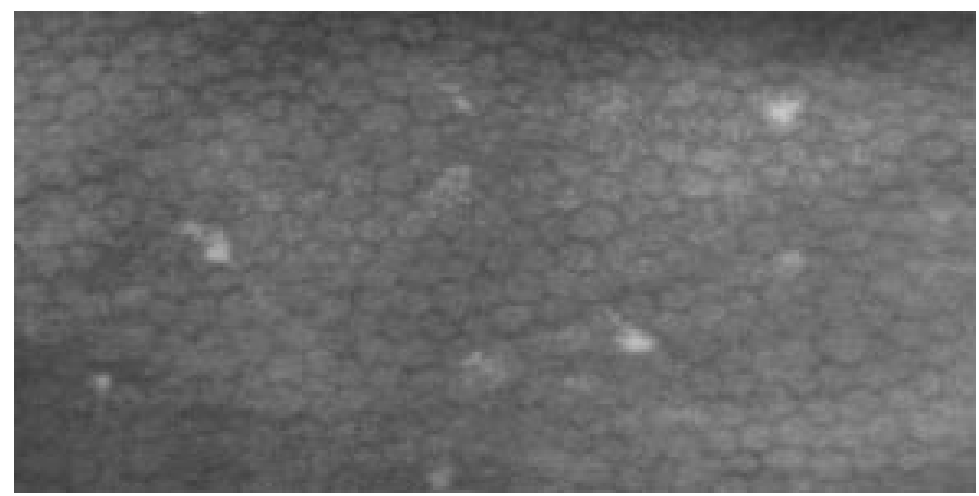

Figure 3 Fine KPs scattered on endothelium.
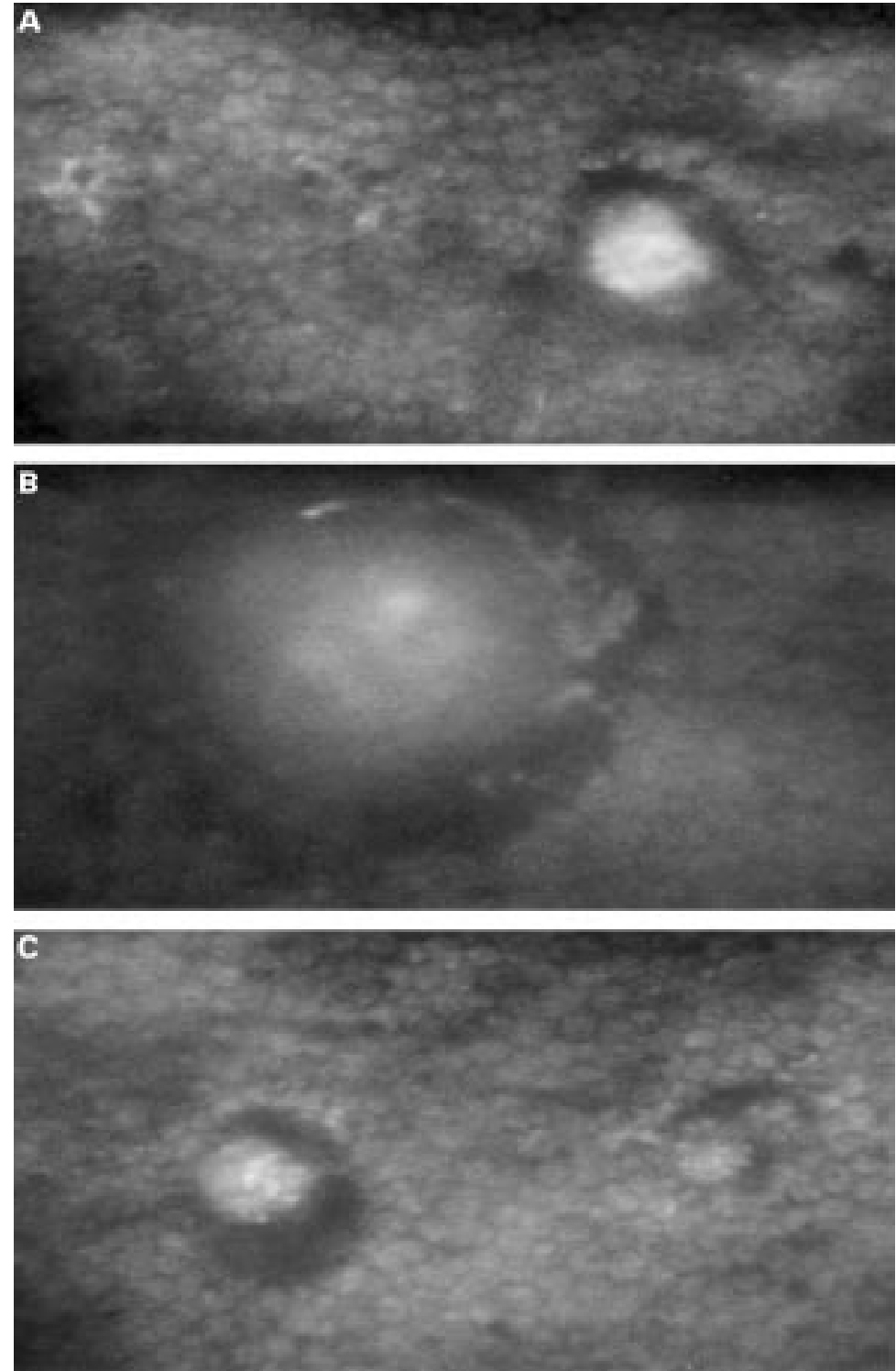

Figure 4 (A) and (B) Resolving KP. Note the appearance of a dark halo surrounding the glistening dense white centre and the gradual increase in the dark shadow in the periphery with the shrinkage of the central white deposit. (C) Old KP. Dark halo surrounding the central white deposit with indistinct cellular margins on resolution of uveitis.

density, and coefficient of variation. Cell size and cell density was calculated in the vicinity of fresh and resolving KP and the average value was taken into consideration. The endothelium of the uninvolved normal eye was analysed simultaneously and was used as a control. Cell density and cell size are summarised in Table 2. At each follow up visit the same procedure was repeated and endothelial images were analysed from the normal eye as well as the eye with uveitis.

\section{STATISTICAL ANALYSIS}

Differences in cell size and cell density were analysed by the Student's $t$ test for paired samples. A $p$ value of $<0.05$ was considered statistically significant. Statistical comparisons were made of the above variables between the endothelium in the vicinity of fresh and resolving $\mathrm{KP}$, fresh $\mathrm{KP}$ and normal endothelium, resolving KP and normal endothelium.

\section{Results}

Thirteen patients were included in the study; their mean age was 49.2 years (range 28-76 years) and there were five females and eight males. All patients had only anterior uveitis except one (50 year old woman) who, in addition, had retinal vasculitis. No underlying systemic aetiology of uveitis was found in any of the patients-two patients had PosnerSchlossman syndrome, one patient had heterochromic cyclitis, one had herpetic uveitis, two patients had idiopathic recurrent iritis, and the rest of the patients had only a first episode of iritis.

\section{FRESH KP}

On specular microscopy, fresh, large or "mutton fat" KP were seen as glistening, dense white deposit with irregular margins. The deposit extended over a round or oval area corresponding to between five and 10 endothelial cells in diameter. The transition between the $\mathrm{KP}$ and the surrounding endothelium was fairly well defined. Vague outline of the cellular constituents of the KP could be visualised in the deposit (Fig 1).

The single KP in Posner-Schlossman syndrome appeared globular occupying more than 15-20 cells diameters on specular microscopy and was actually a conglomeration of three individual $\mathrm{KP}$. The individual $\mathrm{KP}$ was morphologically similar to those of large fresh $\mathrm{KP}$ and the three KP were connected by one or more elongated pseudopodia. Although the margin of the conglomerate was well demarcated from the surrounding endothelium, the transition between the individual $\mathrm{KP}$ was less distinct (Fig 2).

Fresh, small or "fine" KP tended to be more widely distributed in the endothelium and corresponded to only one or two endothelial cells in diameter, sometimes less than one cell in diameter. These too appeared as dense white deposits with well demarcated margins (Fig 3).

RESOLVING KP

On clinical remission of anterior uveitis, small or "fine" KP were seen to disappear without any conspicuous change at the site of original KP. Resolution of large "mutton fat" KP was heralded by the appearance of a dark halo around the shrinking central deposit (Fig 4). 


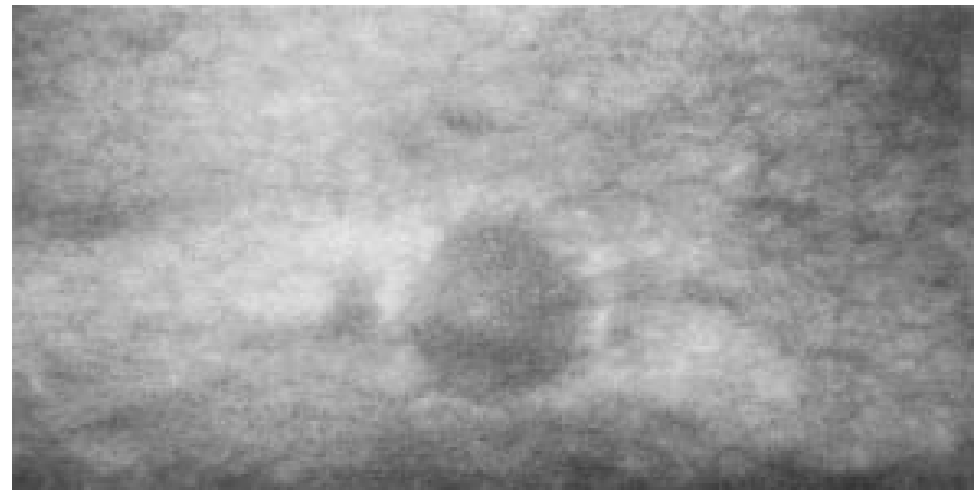

Figure 5 Old KP. Note the dark halo completely replacing the original site of KP.

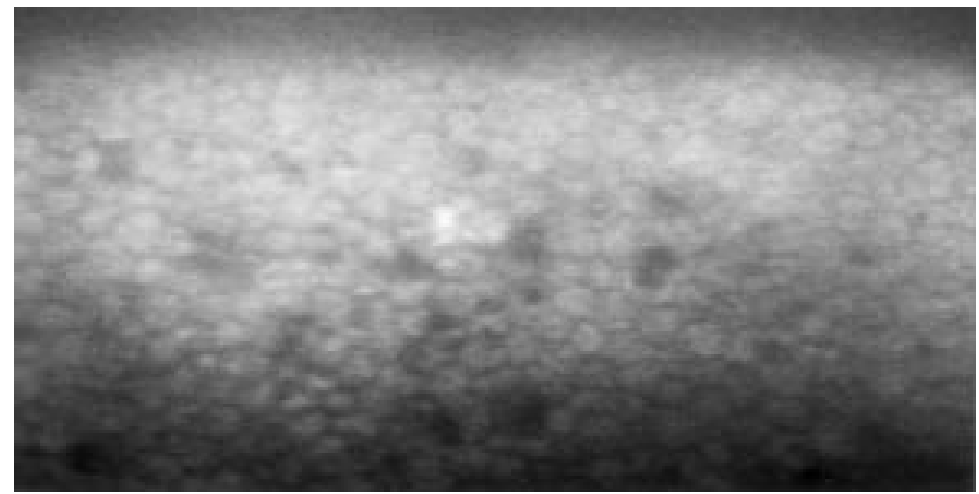

Figure 6 Endothelial blebs observed as dark shadows or defects in endothelium in recurrent uveitis. ing on the endothelium (relief mode, endothelial cell outline is slightly blurred).

ENDOTHELIAL MOSAIC

Endothelial cells in the vicinity of fresh KP had a larger mean cell size and decreased cell density compared with normal endothelium of the opposite eye. This difference was statistically significant $(p=0.002)$. Cell size decreased with corresponding increase in cell density (in the vicinity of $\mathrm{KP}$ ) on resolution of uveitis. Although absolute values did not return to normal in resolving $\mathrm{KP}$, the difference from normal endothelium was not statistically significant.

\section{Discussion}

Morphological changes of endothelium associated with cell loss occur in many conditions including corneal dystrophies, keratoconus, congenital glaucoma, blunt ocular trauma, cataract extraction, and penetrating keratoplasty. ${ }^{13}$ Endothelial abnormalities had been observed in cases of anterior and posterior uveitis. Brooks et al studied the endothelial abnormalities in various corneal disorders ${ }^{13} 14$ and also in uveitis with specular microscopy. To our knowledge, this is the first study to elucidate the different specular microscopic features of KP in different types of uveitis and the distinct morphological changes occurring with resolution of uveitis.

In our study, fresh KP appeared as glistening, dense white deposits and with the clinical remission of uveitis, these deposits appeared less dense and were also smaller in size. A dark halo was noted around the shrinking central white deposit and, eventually, in some instances a dark shadow or defect, corresponding to the original $\mathrm{KP}$, remained as a sequel. The dark shadow or halo observed with the resolution probably indicates degranulation of the precipitate.

The solitary KP observed in PosnerSchlossman syndrome by slit lamp examination was actually a conglomeration of two or three large KP connected by slender pseudopodia which was well demarcated from the normal endothelium. This has not been described before to our knowledge with specular microscopy. Sometimes the distribution of the fine KP were limited to one quadrant of the cornea on slit lamp examination; on specular microscopy they were seen scattered throughout the endothelium and with resolution they were seen to disappear without any sequel. Walter et $a l^{15}$ explained that the fibrin deposition surrounding the inflammatory cells caused the stellate appearance of the KP in cytomegalovirus retinitis and demonstrated the cell pattern of the KP by electron microscopy. Probably the conglomeration of KP observed in Posner-Schlossman syndrome was due to the fibrin deposition around individual KP.

Brooks et $a l^{13}{ }^{14}$ observed single or multiple dark shadows on endothelial mosaic in keratitis and contusion injury and described them as endothelial blebs. Small blebs were considered intracellular or intercellular but the larger ones were almost certainly subendothelial in locaows of an old $\mathrm{KP}$, replacing the fresh $\mathrm{KP}$ on the endothelium, can be seen by slightly defocus- 
tion. On electron microscopy, the larger blebs were found to be an intercellular accumulation of water vacuoles separating the adjacent endothelial cells. These endothelial blebs noted in our study corresponded with the findings of previous authors. We observed these blebs close to the larger KP but not with the fine KP.

These blebs probably indicate endothelial stress and with chronic, recurrent, inflammation corneal decompensation may occur. In our series, in spite of recurrent inflammation and the presence of numerous large and small blebs on the endothelium, none of the patients had corneal decompensation. It could be explained that probably some corneas could withstand the stress better in spite of these blebs.

It is a well established fact that endothelial abnormalities occur in uveitis. ${ }^{4}$ There was a statistically significant difference $(p=0.002)$ in cell size and cell density of endothelium in the vicinity of $\mathrm{KP}$, compared with normal endothelium of the opposite eye. The mean endothelial cell size decreased with a corresponding increase in cell density on resolution of uveitis; the absolute values did not return to normal. There was no statistical significant difference on comparison of mean cell size and cell density of resolving KP with normal endothelium. It might be that endothelial cells surrounding the fresh $\mathrm{KP}$ are larger in size either because of the stress of inflammation or due to some inflammatory mediators acting in an unknown mechanism.

In conclusion, we have described the distinct specular microscopic appearances of KP in different types of uveitis and the changing morphology of the KP with treatment. Significant changes were observed in endothelium in the vicinity of $\mathrm{KP}$ in active uveitis, which returned to near normal values on resolution.
It is not known whether there is any functional significance of the endothelial changes observed.

Some of these data have been presented at the following meetings: ARVO, Fort Lauderdale, Florida, USA, April 1998; Nottingham Eye Research Symposium, Nottingham, UK, January 1999

A Azuara-Blanco was the Vision Express fellow in cornea and contact lenses, University of Nottingham.

1 American Academy of Ophthalmology. Ophthalmic procedures assessment. Corneal endothelial photography. Ophthalmology 1991;98:1464-8.

2 Price NC, Cheng H. Contact and non -contact specular microscopy. Br f Ophthalmol 1981;65:568-74.

3 Koester CJ, Roberts CW, Donn A, et al. Wide field specular microscopy; clinical and research applications. Ophthalmology 1980;81:849-60.

4 Stevenson R, Kirkness CM. A comparison of contact and non-contact specula microscopy in quantifying corneal morphology. Invest Ophthalmol Vis Sci 1994;35(s):1598.

5 Sugar A. Clinical specular microscopy. Surv Ophthalmol 1979;24:21-32.

6 Hartmann C, Koditz W, Automated morphometric endothelial analysis combined with video specular microsdothelial analysis combined

7 Sherrard ES, Buckley RJ. The relief mode. Arch Ophthalmol 1982;100:296-300.

1982;100:296-300.
8 Hartman C, Koditz W. Automated morphometric endothelial analysis combined with video specular microscopy. Cornea 1985;3:155-67.

9 Lester JM, McFarland JL, Bursell SE, et al. Automated morphometric analysis of corneal endothelial cells. Invest Ophthalmol Vis Sci 1981;20:407-10.

10 Olsen T. Changes in the corneal endothelium after acute anterior uveitis as seen with the specular microscope. Acta Ophthalmol 1980;58:250-6.

11 Olsen T. Transient changes in specular appearance of the corneal endothelium and in corneal thickness during anterior uveitis. Acta Ophthalmol 1981;59:100-9.

12 Inomata H, Smesler GK. Fine structural alterations of corInomata $\mathrm{H}$, Smesler GK. Fine structural alterations of corOphthalmol Vis Sci 1970;4:272-85.

13 Brooks AMV, Grant G, Gillies WE. The use of specular microscopy to investigate unusual findings in the corneal endothelium and its adjacent structures. Aust NZ Ophthalmol 1988;16:235-43.

14 Brooks AMV, Grant G, Gillies WE. Differentiation and assessment of corneal endothelial changes associated with diseases of the anterior segment of the eye. Aust NZ $\mathcal{F} O p h$ thalmol 1987;15:65-70.

15 Walter kA, Coulter VL. Corneal endothelial deposits in patients with cytomegalovirus retinitis. Am f Ophthalmol 1996;121:391-6.

16 Rao GN, Stevens RE, Mandelberg AI, et al. Morphological variations in graft endothelium. Arch Ophthalmol 1980;98: 1403-6. 